\title{
Tilted Dirac cone on W(110) protected by mirror symmetry
}

\author{
A. Varykhalov, D. Marchenko, J. Sánchez-Barriga, E. Golias, and O. Rader \\ Helmholtz-Zentrum Berlin für Materialien und Energie, Elektronenspeicherring BESSY II, Albert-Einstein-Str. 15, 12489 Berlin, Germany
}

\author{
G. Bihlmayer \\ Peter Grünberg Institut and Institute for Advanced Simulation, Forschungszentrum Jülich and JARA, 52425 Jülich, Germany
}

(Received 8 May 2016; revised manuscript received 18 May 2017; published 20 June 2017)

\begin{abstract}
Topologically nontrivial states reveal themselves in strongly spin-orbit coupled systems by Dirac cones. However, their appearance is not a sufficient criterion for a topological phase. In topological insulators, where these states protect surface metallicity, they are straightforwardly assigned based on bulk-boundary correspondence. On metals, where these states are suspected to have tremendous impact as well, e.g., in catalysis, their topological protection is difficult to assess due to the lacking band gap and the frequent assignment to topological properties appears unjustified. Here, we discover by angle-resolved photoemission a state with the dispersion of a Dirac cone at a low-symmetry point of W(110). Our ab initio calculations predict this feature with a linear band crossing and high spin polarization. However, instead of being born by topology, the states arise from Rashba split bands and do not fundamentally depend on the opening of a spin-orbit gap. On the other hand, we find that the [001] mirror plane protects the band crossing point and renormalizes the dispersion towards a Dirac-cone shape. In this sense, the discovered state is the metal counterpart of the surface state of a topological crystalline insulator. The Dirac cone is tilted due to its origin in an accidental band crossing away from high symmetry points. Tilted Dirac cones have recently been predicted for two- and three-dimensional materials and were observed in three-dimensional Weyl semimetals. Accordingly, the protection and renormalization by mirror symmetry uncovered here are a potentially much wider spread phenomenon which does not require topological properties. Our results also indicate why the massive gapless crossing predicted for topological crystalline insulators has never been observed.
\end{abstract}

DOI: 10.1103/PhysRevB.95.245421

\section{INTRODUCTION}

Topological insulators possess surface states due to a bulk-boundary correspondence. They are protected by time-reversal or crystal symmetries and form in most cases the quasirelativistic linear dispersion of Dirac cones across the absolute band gap of the volume [1-4]. The identification of topologically nontrivial states has been achieved on the one hand based on the direct measurement of these Dirac-like dispersions and on the other hand by the evaluation of the Chern numbers for time-reversal-invariant $\mathbb{Z}_{2}$ topological insulators and mirror-symmetry-protected topological crystalline insulators [5-8]. The latter is facilitated by the presence of an absolute band gap. In this case there are rules that, for inversion-symmetric systems, allow for a simple counting of parities of electronic states at high-symmetry points in reciprocal space for occupied states up to the band gap [9] or a k-space integral of all occupied bands [10].

In a metal system, however, this analysis is not feasible because such procedures are ill defined. In fact, $\mathrm{Sb}$, which has early on been suggested as a semimetal with topological surface state [11] due to its similarity with $\mathrm{Bi}_{0.9} \mathrm{Sb}_{0.1}$ $[11,12]$ has been analyzed in terms of topological indices (permitted due to a relative gap) but the possibilities to predict topologically protected surface states in a rigorous way turned out to be rather limited due to the bulk pockets at the Fermi energy [13]. The semimetal Bi can be distorted to a $\mathbb{Z}_{2}$ phase [14] and metastable crystalline topological insulator phase of $\mathrm{Bi}$ was recently predicted [15]. We have previously suggested a surface state with a giant Rashba splitting on $\operatorname{Ir}(111)$ to be of topological origin based on its connection to the bulk states [16] as well as an off-center surface state on $\mathrm{Sb}_{2} \mathrm{Te}_{3}$ [17]. This state is completely occupied but is found to satisfy the conditions for surface state formation due to a spin-orbit gap $[17,18]$.

Without the need to obey the restrictions of Lorentz invariance and particle-hole symmetry, Dirac cones in condensed matter may develop anisotropy and/or tilt. Tilted Dirac cones in two dimensions have been found in the theoretical description of two-dimensional organic superconductors [19,20] and in strained graphene [20-22]. These are expected to lead to remarkable effects in transport such as a change of the Fano factor [23] and a light-induced valley current [24] but have not been observed yet directly. Three-dimensional topologically protected tilted Dirac cones appear in Weyl semimetals, in particular cases the tilt is so large that the upper Dirac cone lies partly below the energy of the Dirac point such as in $\mathrm{WTe}_{2}$ [25].

At W(110), a spin-orbit-split and spin-polarized surface state has been identified previously [26,27]. For this state, the shape of a Dirac cone with corresponding spin texture centered about the $\bar{\Gamma}$ point of the surface Brillouin zone has independently been identified by two groups but with differing interpretations [28,29]. A Dirac cone was seen but with different group velocities in the (110) surface plane [30]. Although no direct connection with the bulk topology could be established, the similarity to three-dimensional topological insulators was pointed out [29], and the state was most recently called "pseudotopological surface state" [31]. On the other hand, it was shown that the band connectivity and spin structure for pure W(110) changes after deposition of already 1 monoatomic layer of $\mathrm{Al}$ rather indicating a nontopological character [28]. In that case, the spin texture was explained as being due to Rashba polarization [28], i.e., the combined effects of spin-orbit interaction and broken inversion symmetry [32,33]. Previously, the deposition of $\mathrm{Au}, \mathrm{Ag}$, and $\mathrm{Cu}$ 

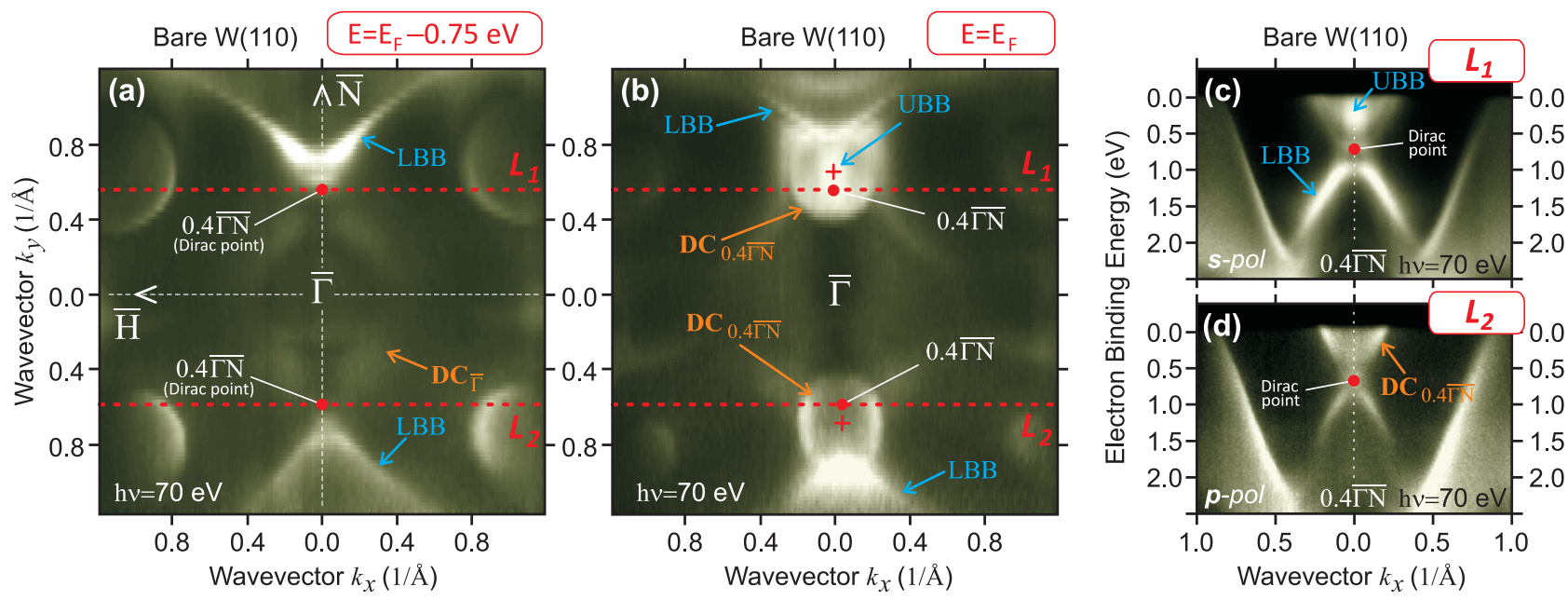

FIG. 1. Electronic structure of bare $\mathrm{W}(110)$ measured at $\mathrm{h} v=70 \mathrm{eV}$; (a) constant energy surface sampled at binding energy of $0.75 \mathrm{eV}$; (b) Fermi surface. Note the elliptical orbits denoted as $\mathrm{DC}_{0.4 \overline{\Gamma \mathrm{N}}}$, which occur at about $40 \%$ of the $\overline{\Gamma \mathrm{N}}$ distance. Centers of these orbits (red crosses) are displaced from their points of convergence $0.4 \overline{\Gamma \mathrm{N}}$ at $E_{B}=E_{F}-0.75 \mathrm{eV}$ (red dots) thus indicating tilted Dirac cones. (c) Dispersion of the $\mathrm{DC}_{0.4 \overline{\Gamma N}}$ band measured at more $s$-polarized light (enhanced signal from $d_{x^{2}-y^{2}}$ orbitals) and traced along the $\mathrm{L}_{1}$ line. (d) Dispersion of $\mathrm{DC}_{0.4 \overline{\Gamma \mathrm{N}}}$ measured at more $p$-polarized light (enhanced signal from $\mathrm{d}_{z^{2}}$ orbitals) and traced along the $\mathrm{L}_{2}$ line.

monolayers on $\mathrm{W}(110)$ has been used to distinguish between the roles of the spin-orbit splitting at the surface and in the volume. The monolayers modify the spin-dependent surface states of the W(110) by hybridization with noble-metal $s$ and $p$ states [34,35]. Recent calculations of W, where artificial strain opens semimetal band gaps that allow to compute Chern numbers, find nontrivial bulk band structures in some case [36]. In that work, similar features are reported for $\mathrm{Mo}, \mathrm{Nb}$, and Ta as well.

Here, we discover by angle-resolved photoemission an unexpected surface state along a high-symmetry direction of the surface Brillouin zone but away from a high-symmetry point. The linear dispersion at the crossing point is seen directly. Constant-energy maps demonstrate also the elliptical, almost circular shape in momentum space and its tilt. Nevertheless, we argue that the state is a trivial surface state and suggest how trivial bands can generally acquire the shape of a Dirac cone.

\section{METHODS}

The W(110) sample was cleaned as described elsewhere [37]. Cleanliness of the bare W(110) surface was verified by the presence of an intense surface-derived component of the $\mathrm{W} 4 f$ core level [38]. In experiments with $\mathrm{Au} / \mathrm{W}(110)$ the amount of deposited $\mathrm{Au}$ was determined from a careful calibration of the evaporation rate by a quartz microbalance. After the deposition, the sample was annealed at $\sim 700 \mathrm{~K}$. Our scanning tunneling microscopy characterizations of annealed samples reveal that Au forms on W(110) continuous monolayers. Full monolayers of $\mathrm{Au}$ were also giving sharp low-energy electron diffraction patterns hence indicating high structural quality of the adlayers.

Photoemission experiments have been performed at synchrotron BESSY II with the endstations ARPES $1^{2}$ and RGBL2 equipped with Scienta R8000 and Scienta R4000 analyzers, respectively. The endstation has been installed at the beamlines UE112-PGM2 and UE112-PGM1. Different photon energies varying between 20 and $80 \mathrm{eV}$ have been used for the charac- terization of the band structure. The polarization of light was linear and selectable between $s$ or mixed $(s+p)$. A scheme of the photoemission experiment is shown in Fig. 1 of Ref. [39].

Density-functional-theory calculations of the band structure have been conducted in the generalized gradient approximation [40] using the full-potential linearized augmented plane-wave method in film geometry [41] as implemented in the FLEUR code (http://www.flapw.de) with accounting for spin-orbit interactions. The W(110) surface was realized as a 26-monolayer-thick slab. The topmost few layers of $\mathrm{W}$ have been relaxed (more detailed information can be found in Ref. [39]). Spin-orbit interaction is treated self-consistently as described in Ref. [42].

\section{RESULTS}

\section{A. Photoemission evidence for the Dirac-like state off-centered from $\bar{\Gamma}$}

The angle-resolved photoemission (ARPES) data in Fig. 1(a) represent a constant-energy surface of W(110) sampled at $0.75 \mathrm{eV}$ below the Fermi level. The rhombic contour $\mathrm{DC}_{\bar{\Gamma}}$ appearing around the center of the surface Brillouin zone $\bar{\Gamma}$ belongs to the recently discovered Dirac-cone-like surface resonance mentioned in the introduction [28,29]. When we inspect the measured constant energy surfaces along the vertical direction [defined as $k_{y}$ in Fig. 2(f)] for various binding energies between $0.75 \mathrm{eV}$ and $E_{F}$ we find two other contours occurring at about $40 \%$ of the distance from $\bar{\Gamma}$ to the zone boundary at $\overline{\mathrm{N}}$ and evolving from points at $E_{B}=E_{F}-0.75 \mathrm{eV}$ (a red dot in Fig. 1) toward elliptical orbits at the Fermi surface [Fig. 1(b)]. The evolution of these contours with binding energy reveals the presence of conelike bands (precise position of which will be determined below with help of theory as $0.38 \overline{\Gamma N}$ ). These contours, denoted as $\mathrm{DC}_{0.4 \bar{\Gamma} \mathrm{N}}$, and their surroundings appear differently in the upper and the lower part of Fig. 1(b). This is related to photoemission matrix element effects due to different relative weights of 

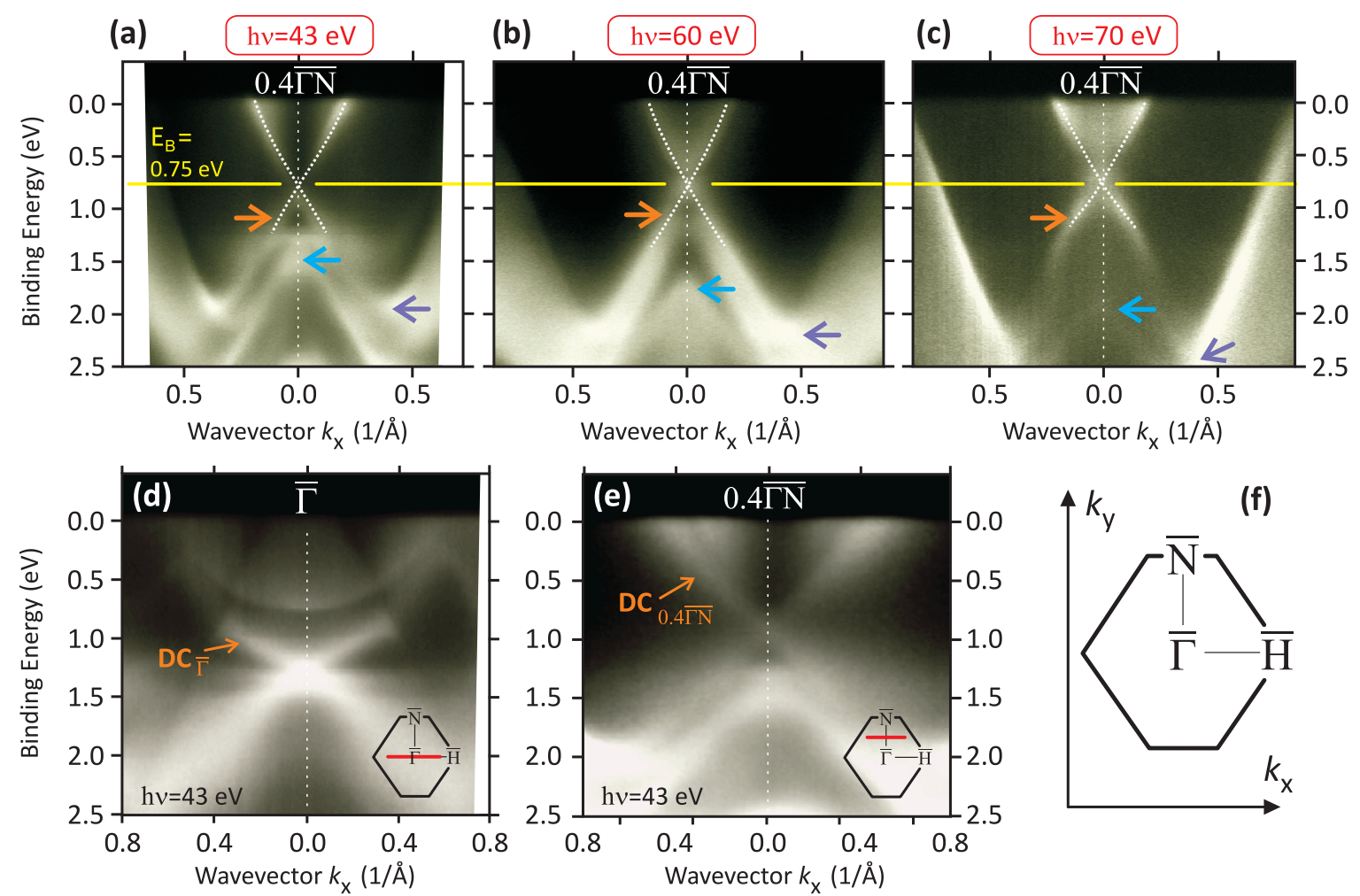

FIG. 2. Two-dimensional character of the $\mathrm{DC}_{0.4 \overline{\Gamma \mathrm{N}}}$ band. Identical dispersion with $\mathbf{k}_{\|}\left(k_{x}\right)$ at photon energies (a) $h v=43$, (b) 60 , and (c) $70 \mathrm{eV}$ reveals the absence of dispersion with $\mathbf{k}_{\perp}$ and the $2 \mathrm{D}$ character of a surface resonance. In contrast, the bulk bands (color arrows) disperse strongly. (d) and (e) Comparison of (d) the established surface resonance at $\bar{\Gamma}$ and (e) the new one reported here at $0.4 \bar{\Gamma} \mathrm{N}$. Directions of measurements as denoted in the insets (red line). Photon energy $h v=43 \mathrm{eV}$. (f) Sketch defining directions of $k_{x}$ and $k_{y}$ wave vectors relative to the W(110) surface Brillouin zone.

$s$ - and $p$-polarized light. The effect occurs as a result of changing angle of the light polarization vector during the band structure mapping by scanning the polar angle $\Theta$ of the sample (see Fig. 1 of Ref. [39]). We will use further below these differences for identification of the states.

The Fermi surface in Fig. 1(b) is particularly remarkable. An intense signal from the elliptical contours appears to be centered not around their points of convergence at $E_{B}=E_{F}-0.75 \mathrm{eV}(0.4 \overline{\Gamma \mathrm{N}})$, but more far away from $\bar{\Gamma}$. Centers of these elliptical orbits [red crosses in Fig. 1(b)] are displaced from $0.4 \overline{\Gamma \bar{N}}$ by $\Delta k_{y}=(0.09 \pm 0.03) \AA^{-1}$ toward the Brillouin zone boundary at $\bar{N}$. This in turn indicates that the conelike bands $\mathrm{DC}_{0.4 \overline{\Gamma \mathrm{N}}}$ are asymmetric and tilted in $\mathbf{k}$ space.

Figures 1(c) and 1(d) reveal precise dispersions of $\mathrm{DC}_{0.4 \bar{\Gamma}}$ bands, measured through $0.4 \overline{\Gamma \mathrm{N}}$ and perpendicular to the $\overline{\Gamma \mathrm{N}}$ direction [the traces are labeled $L_{1}$ and $L_{2}$ in Fig. 1]. A Diraccone-like linear dispersion is seen in both panels along with upper and lower bulk bands (UBB and LBB, respectively) which are contributing substantial photoemission signal. In view of the prediction of a large number of 3D Dirac and Weyl cones in metals such as Fe [43], we need to experimentally determine the dimensionality of the feature. The distinction between bulk and surface bands has been made on the basis of their dispersion with the electron wave vector perpendicular to the surface, $\mathbf{k}_{\perp}$. This is achieved by variation of the photon energy as shown in Fig. 2 [44,45]. In total, we have tested photon energies from 20 to $80 \mathrm{eV}$ [selected results are reported in Figs. 2(a)-2(c)] and found basically no change in the binding energy of the state. This identifies a two-dimensional Diraccone-like state, and, in particular, the energy of the crossing point $(-0.75 \mathrm{eV})$ remains constant $\pm 50 \mathrm{meV}$, which is within the experimental error.

Note that the lower bulk band LBB is not symmetric around $0.4 \bar{\Gamma} \mathrm{N}$ and, instead, disperses from $0.4 \overline{\Gamma \mathrm{N}}$ towards $\overline{\mathrm{N}}$ steeply upward. This is, e.g., seen from the comparison of the constant energy surfaces in Figs. 1(a) and 1(b).

Figures 2(d) and 2(e) emphasize the dispersion of the new surface resonance at $0.4 \overline{\Gamma \mathrm{N}}$ reported here, by comparing it to the established surface resonance at $\bar{\Gamma}$. Directions of ARPES cuts are shown in the insets by red line.

\section{B. First-principles calculations of $\mathbf{W}(\mathbf{1 1 0})$}

In a first step, the projection of the $\mathrm{W}$ bulk band structure on the (110) surface has been calculated (see Fig. 2 of Ref. [39]). This is required to clarify if a spin-orbit-induced bulk band gap is present where the measured Dirac-cone candidate is situated. In a second step, the W(110) surface has been modeled by a symmetric slab. Figure 3 shows for 26 atomic layers of $\mathrm{W}$ a similar cut as $L_{1}$ in Fig. 1(c). The cut was chosen at the smallest value of the bulk band gap which, after refinement, is at $0.38 \overline{\Gamma N}$ (for neighboring cuts see Fig. 3 of Ref. [39]) There are two remarkable observations from Figs. 3(a) and 

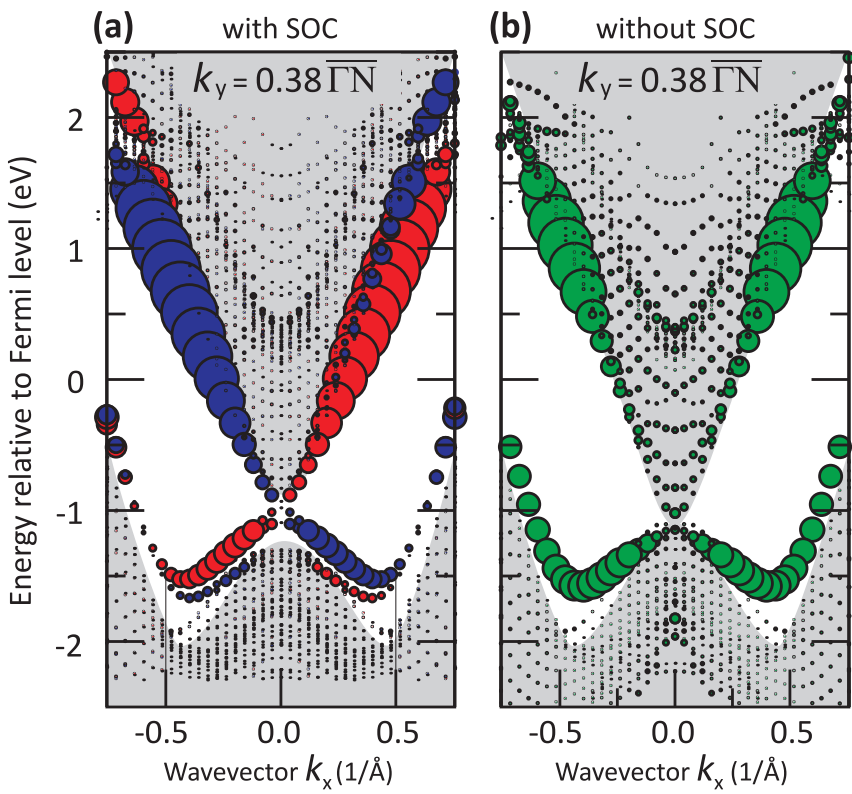

FIG. 3. Band structure of the W(110) surface calculated offcenter of the surface Brillouin zone. Wave vector $k_{x}$ runs perpendicular to $\overline{\Gamma N}$, and $k_{x}=0$ corresponds to the point of minimum band gap between two surface states (at $k_{y}=0.38 \overline{\Gamma \mathrm{N}}$ ). (a) For spinorbit coupling included a surface state with quasilinear dispersion appears which is largely degenerate with the upper bulk band (surface resonance). Colors denote the directions of spin parallel to $k_{y}$ direction. The size of the markers is proportional to the spin-polarization of the states. (b) For a calculation without spin-orbit interaction, the bulk band gap closes as seen at $k_{x}=0$. Nevertheless, the surface state (surface localization is indicated by the size of the green markers) does not disappear. This behavior demonstrates that the origin of these states is not a topology change induced by spin-orbit effects. The thickness of W slab is 26 atomic layers, and the bulk projected states are displayed as well (gray shaded).

3(b). Firstly, in Fig. 3(a), a linear, quasirelativistic dispersion is indeed obtained for a two-dimensional surface-localized state (surface spin polarization indicated by symbol size). The state is highly spin polarized as seen from the figure. The colors denote the spin projection parallel to $k_{y}$ (i.e., parallel to $\overline{\Gamma N}$ ), hence the spin texture is indeed that typical of a Dirac cone with spin locked perpendicular to the linear momentum. However, since the cone is not centered at $\bar{\Gamma}$, this polarization component decreases with $k_{x}$ and vanishes at $k_{x}=0$.

Figure 3(b) shows the same calculation but without spinorbit interaction. Here, the bulk band gap has closed at $k_{x}=0$. Here, the size of the symbols indicates the surface character. From a surface state that is created solely by the bulk-boundary correspondence of a topological insulator where the band-inversion is created by spin-orbit coupling, we expect that it disappears when the spin-orbit gap closes. This is the scenario known from the $\mathrm{Bi}_{2} \mathrm{Se}_{3}$ family of compounds. A comparison of $\mathrm{Sb}_{2} \mathrm{Se}_{3}, \mathrm{Sb}_{2} \mathrm{Te}_{3}, \mathrm{Bi}_{2} \mathrm{Se}_{3}$, and $\mathrm{Bi}_{2} \mathrm{Te}_{3}$ showed that for $\mathrm{Sb}_{2} \mathrm{Se}_{3}$ with the lowest spin-orbit interaction the Dirac-cone surface state is in fact absent whereas the remaining three compounds feature prominent topological surface states [46]. The persistence of the present state in the nonrelativistic calculation tells us that there is no one-to-one relationship between the observed surface state and possible spin-orbit induced band-inversions in the bulk band structure. It is also important to mention that the counterpart of the present surface state was recently observed on Mo(110) [47] where the spin-orbit interaction is strongly reduced as compared to $\mathrm{W}$. This is consistent with the picture that spin-orbit effects are not at the origin of the observed Dirac-cone shaped states.

From the calculation of Fig. 3 it cannot be judged whether or not the linear dispersion is really gapless. The obstacle is the surface-surface interaction as artifact of the symmetric slab geometry which, in the present case of a 26 layer geometry, leaves a gap at the crossing point of about $30 \mathrm{meV}$. Therefore an asymmetric slab with an additional monolayer $\mathrm{Au}$ on one side has been calculated as well. The states on the $\mathrm{W}$ and the $\mathrm{Au}$ side, respectively, are identified by their surface localization, as usual. Figure 4(a) shows the $\overline{\Gamma N}$ direction where the intersection from Fig. 3 appears at $k_{y}=0.55 \AA^{-1}$ (corresponds to $0.38 \overline{\Gamma \mathrm{N}}$ ). The bands cross due to their opposite spin direction as indicated by red and blue colors. Note that perpendicular to $\overline{\Gamma N}$ a linear crossing is not necessarily expected, nevertheless Fig. 4(b) shows that the bands are spin polarized and nondegenerate and that they cross each other with amazingly linear dispersion behavior. The additional degeneracy of the Dirac cone locally seen near the touching point in Fig. 3(a) (and in inset to Fig. 3 of Ref. [39]) is also related to the symmetry of the tungsten slab possessing two equivalent surfaces. This is not the case with Au-terminated W(110) (Fig. 4) for which such equivalence is broken due to the Au coverage on one of the surfaces.

\section{Comparison to Au monolayer on W(110)}

We mentioned above that the bands forming Dirac-conelike state in question are present also in the nonrelativistic calculations of W(110) without spin-orbit interaction. In the relativistic calculations, the degeneracy of the bands is lifted by a Rashba effect but for some momentum vectors the spin-split branch is shifted into the bulk projection and broadened so that the splitting is apparently too small to be resolved in experiment (see Fig. 3). The Rashba spin-orbit effect [32,33], on the other hand, leads in the calculation to a high spin polarization of the other branch which remains in the gap. Therefore, a spin-resolved ARPES experiment would not give the decisive answer as to the topological character of this Dirac-cone-like state. On the surface covered with an atomic layer of $\mathrm{Au}$, however, a large splitting occurs. This is seen from Figs. 4(c) and 4(d) (more details about the structure can be found in Ref. [39]). Here, the symbol sizes and colors relate to the surface localization and spin polarization, respectively, at the Au terminated side (note that both $\mathrm{W}$ and Au terminated surfaces contribute in the same slab). Figure 4 in Ref. [39] shows the Au termination calculated without spin-orbit interaction where it can be confirmed that the number of states remains again identical, taking into account the degeneracy lifted by the Rashba effect in Figs. 4(c) and 4(d). These bands should be straightforward to observe in the experiment. In fact, Fig. 5 shows the experimental data for 1 atomic layer of $\mathrm{Au} / \mathrm{W}(110)$. From Ref. [48] we know that this system grows in the Nishiyama-Wassermann orientation 

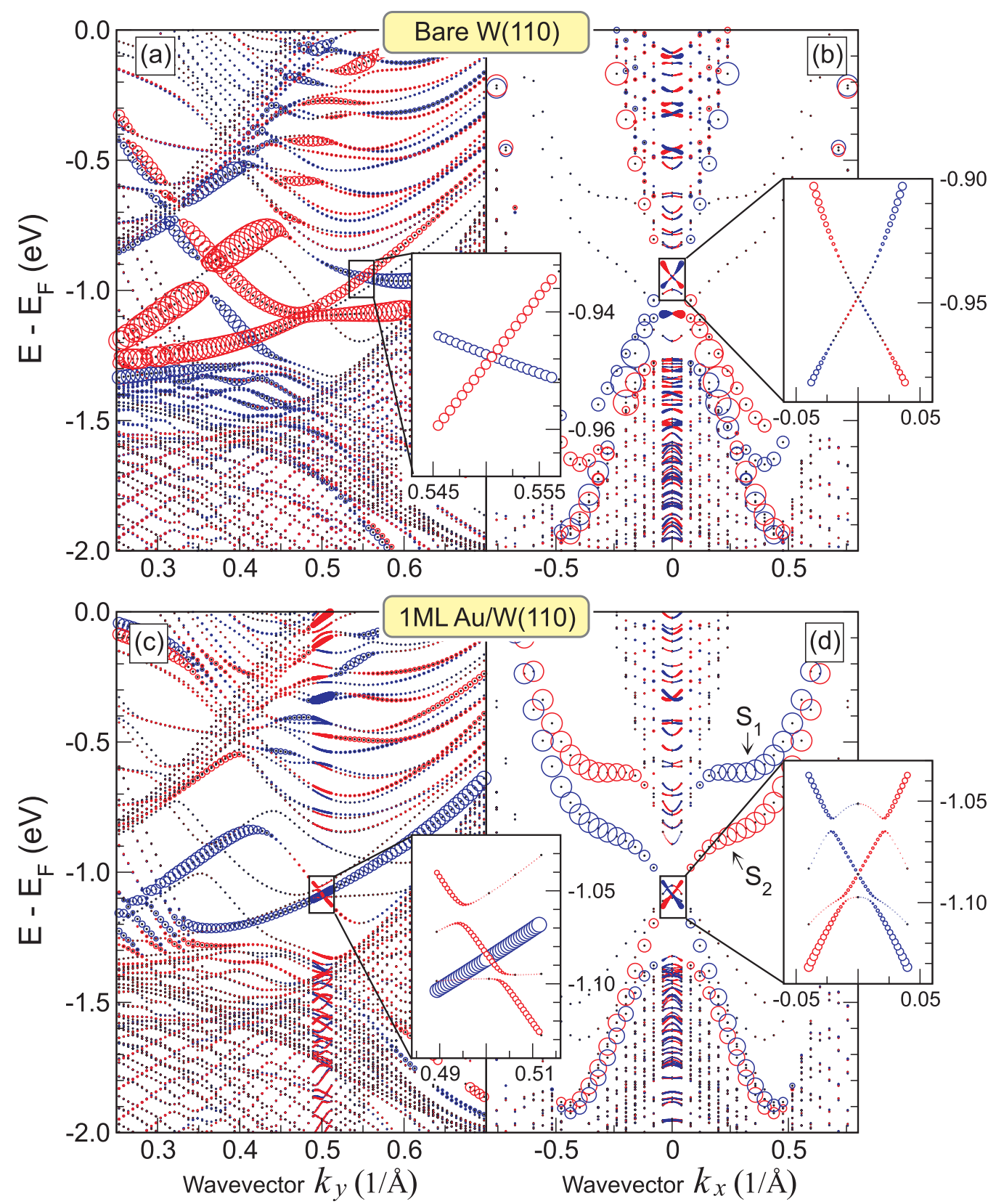

FIG. 4. Slab calculation of W(110) with a single atomic layer of Au on one side. (a) Band structure along $\overline{\Gamma N}$ direction of the surface Brillouin zone $\left(k_{y}=0.55 \AA^{-1}\right.$ corresponds to $\left.0.38 \overline{\Gamma \mathrm{N}}\right)$ with spin-polarization localized at the clean W(110) side marked with red and blue colors. Along $\overline{\Gamma \bar{N}}$ several spin polarized bands cross. (b) At the crossing point (enlarged by the inset) the bands also cross with linear dispersion in the perpendicular to $\overline{\Gamma \bar{N}}$ direction (direction of $k_{x}$ ). (c) For the Au-terminated side, the band crossing shifts towards $0.5 \AA^{-1}$. (d) This means higher binding energy for the crossing which remains linear both along the $k_{x}$ and $k_{y}$ direction. Spin-orbit split bands appear at binding energies $0.5-1.0 \mathrm{eV}$.

that does not break the mirror symmetry along $\overline{\Gamma \bar{N}}$. Fig. 5(a) shows a constant energy surface traced at $E_{B}=E_{F}-0.3 \mathrm{eV}$. Figure 5(d) displays ARPES cuts through $0.4 \overline{\Gamma N}$ compared vis-à-vis the measurement of bare W(110) [Fig. 5(e)]. The extra splitting from Fig. 4 is perfectly reproduced in the experimental data (marks $S_{1}$ and $S_{2}$ ). For comparison, also the $\bar{\Gamma}$ state is shown [Figs. 5(b) and 5(c)]. For the $\bar{\Gamma}$ state, it was already known from measurements along $\overline{\Gamma S}$ that the large spin-orbit splitting of Au on W(110) disappears on a Mo(110) substrate with reduced spin-orbit interaction [34], i.e., that the spin-orbit split states are a property of the substrate. This agrees with the conclusions from the present calculations without spin-orbit interactions.

\section{Formation of the Dirac-cone shape and protection by mirror symmetry}

The question to resolve is the contrasting indications in favor and against a topological character and protection of the observed Dirac-cone-like dispersion. Figure 4 in Ref. [39] 

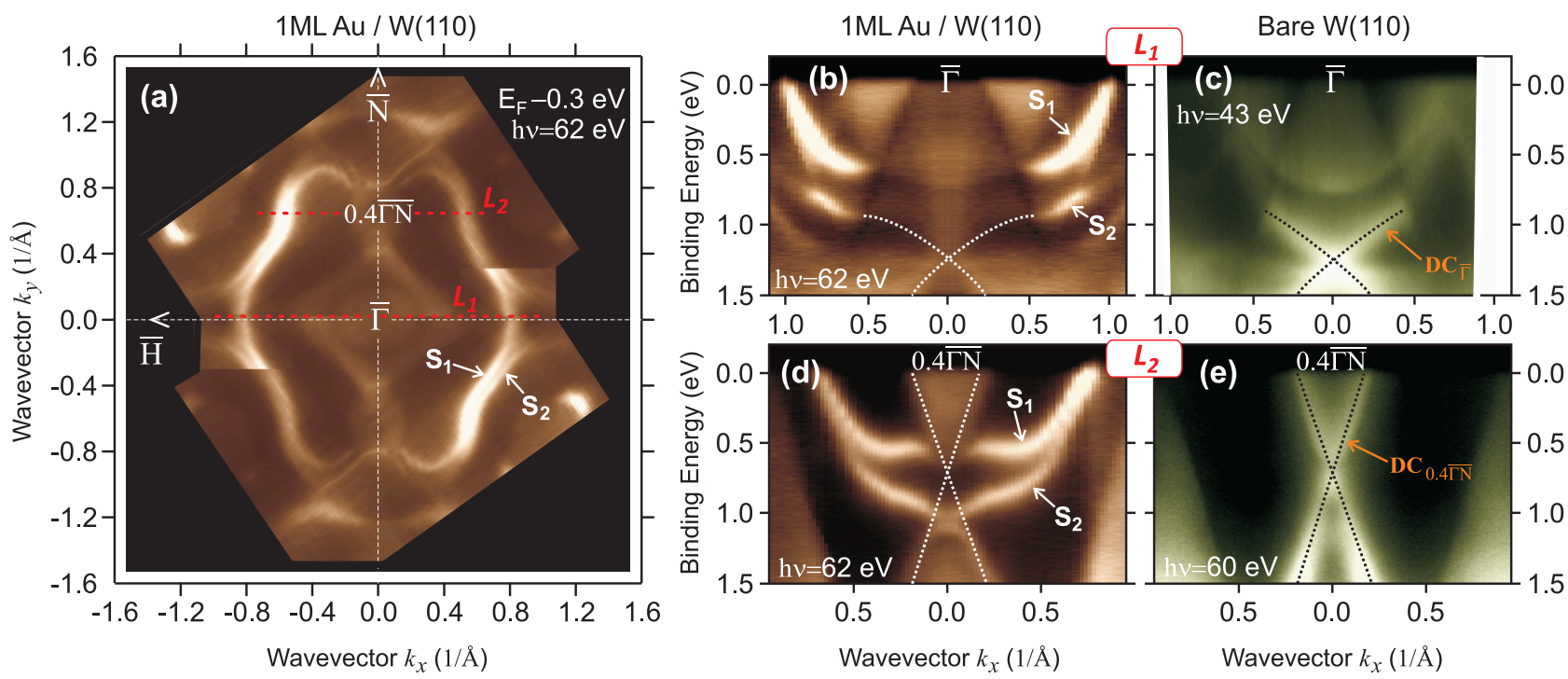

FIG. 5. (a) Band mapping and (b) and (d) dispersions of $1 \mathrm{ML} \mathrm{Au} / \mathrm{W}(110)$ as compared to (c) and (e) the pure W(110). (a) A new two-dimensional Fermi surface (the image shows constant energy surface at $E_{B}=E_{F}-0.3 \mathrm{eV}$ ) with (b) and (d) new splittings in the dispersion appears. Near $0.4 \overline{\Gamma N}$ the crossing at higher binding energies is inherited from the original crossing in $\mathrm{W}(110)$.

shows the nonrelativistic version of Figs. 4(c) and 4(d). In Fig. 4 of Ref. [39], the bands are easier to follow without spin-orbit splitting, and the orbital character has been analyzed and is indicated in the figure. According to these results, the bands which cross do not only differ in spin but also in orbital character. This is confirmed by the experiment: it agrees with the different intensity behavior of the upper and lower cones when the light polarization is varied in the ARPES experiment [see Figs. 1(b)-1(d)]. The calculation obtains the upper band as $d_{x^{2}-y^{2}}$ and the lower one as $d_{z^{2}}$. Along $k_{x}$, these bands should have a parabolic dispersion and not a Dirac cone.

In Fig. 6, we present a model explaining how the band dispersion is renormalized due to the spin to mimic the formation of a Dirac cone. An upward dispersing massive (convex) band and a downward dispersing (concave) band are expected in view of the substantial spin-orbit interaction in $\mathrm{W}$ to be Rashba split [Fig. 6(a)]. For simplicity, we assume that the bulk band projection leaves a certain $\mathbf{k}$-space range (white rhombus in Fig. 6) for the formation of well localized two-dimensional states so that we can concentrate on one spinsplit branch. The crossing point along $k_{y}$ [zoomed in Fig. 6(b)] should give rise to the touching of massive parabolas expected in the perpendicular direction. For smaller $k_{y}$ than the touching point they form a gap. For larger $k_{y}$ beyond the touching point, the spin texture demands a certain hybridization which opens a band gap everywhere except near $0.38 \overline{\Gamma N}$.

The last step to produce the cone is to convert the parabolic touching in Fig. 6(c), panel 2, to a linear crossing. This needs a certain interaction between the two parabolas and for this they must have different curvature, as is assumed in the model in Fig. 6(d). It also requires a noncircular energy surface as is typical for a (110) surface with $C_{2}$ symmetry. At $k_{x}=0$, the mirror plane, obviously the spins point in exactly opposite direction. But away from the touching point, an interaction occurs because the spins of the two parabolas for the same $k_{x}$ form a finite angle. This interaction leads to a renormalization $\delta$ of the band dispersion, which we approximate by the overlap integral $\left\langle\phi_{2} \mid \phi_{1}\right\rangle$ between spin wave functions $\phi_{1}$ and $\phi_{2}$ and is displayed in Fig. 6(e). $\delta$ increases linearly with $k_{x}$ as follows from geometric considerations (see Fig. 5 in Ref. [39]). The renormalization affects the dispersions in Fig. 6(d) along $k_{x}$ in a linear-combination-of-atomic-orbitals-like ansatz according to

$$
\begin{aligned}
E_{\mathrm{a}}^{\prime} & =\left(E_{\mathrm{a}}+E_{\mathrm{b}}\right) / 2+\sqrt{\left(E_{\mathrm{a}}-E_{\mathrm{b}}\right)^{2} / 4+\delta^{2}} \\
\text { and } \quad E_{\mathrm{b}}^{\prime} & =\left(E_{\mathrm{a}}+E_{\mathrm{b}}\right) / 2-\sqrt{\left(E_{\mathrm{a}}-E_{\mathrm{b}}\right)^{2} / 4+\delta^{2}},
\end{aligned}
$$

which is displayed in Fig. 6(f). The linear interaction term outweighs the quadratic one for small $k_{x}$ and, therefore, a linear crossing appears, and the renormalized dispersion has in fact the shape of a distorted Dirac cone. This is seen in Fig. 6(g) based on our simple model. In general and in the density functional calculations of Fig. 4, the cone is additionally tilted. Note that the mirror plane not only leads to the linear renormalization but also allows the bands to cross because the antiparallel spin alignment cannot open a gap there. The effect of breaking this symmetry and the resulting gap opening is shown in Fig. 6 of Ref. [39] for the Au covered W(110) surface. Our conclusions also apply to the states observed in the independent work of Ref. [31] which appear on the same mirror plane along $\overline{\Gamma \bar{N}}$ and lead to a measured spin texture similar to the one predicted here. For a detailed comparison see Fig. 7 in Ref. [39]. Essential in the present model is the mirror plane. We used in our simple model the spin because it explains also the calculated spin texture in Figs. 4(b) and 4(d). A similar interaction can arise from spinless orbitals where a mirror plane can also protect the crossing and inhibit an interaction which becomes effective away from the mirror plane. We believe that this interaction which does not require a spin-orbit gap or topologically nontrivial character but nevertheless creates a Dirac cone shape and protects the crossing point, applies more generally than in our present example. 

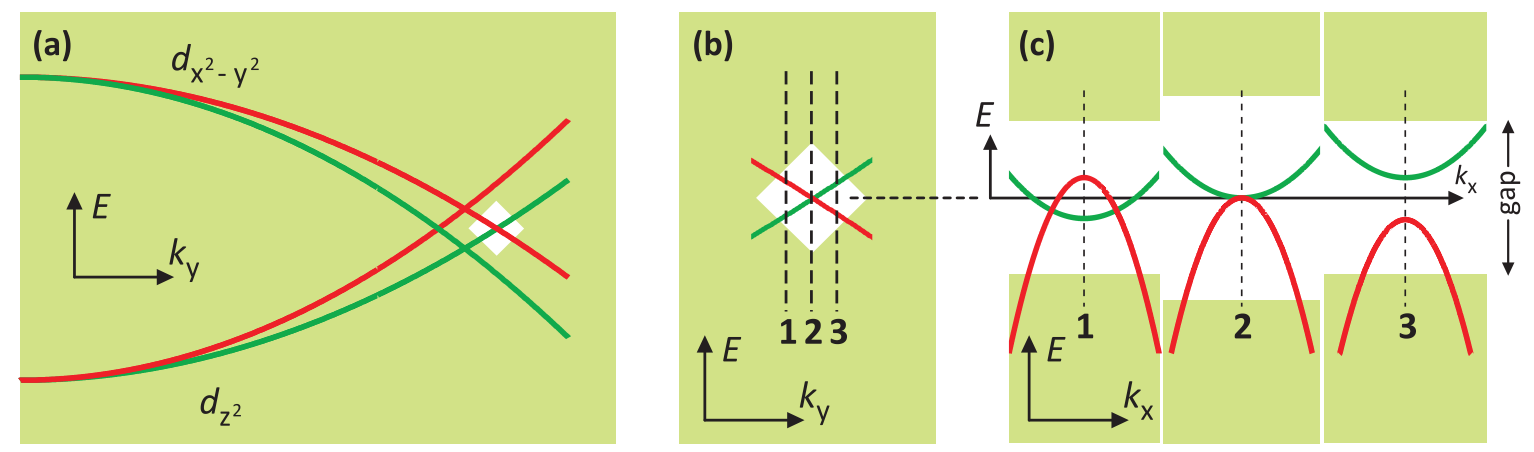

(d)

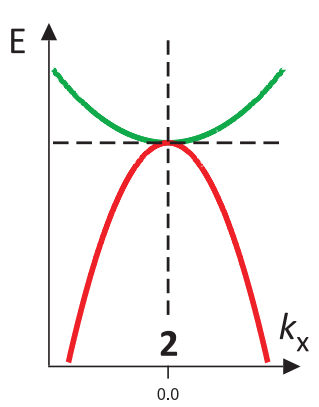

(e)

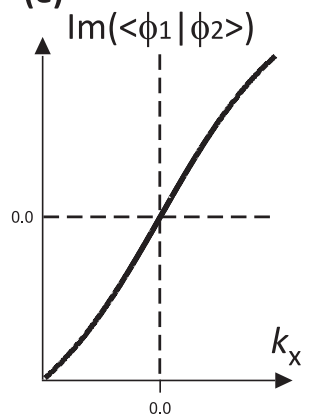

(f)

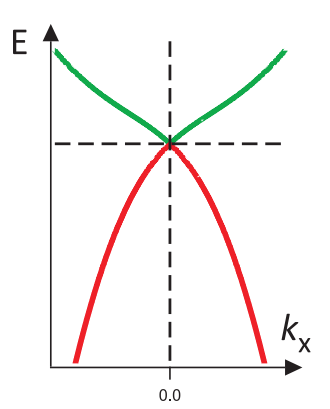

(g)

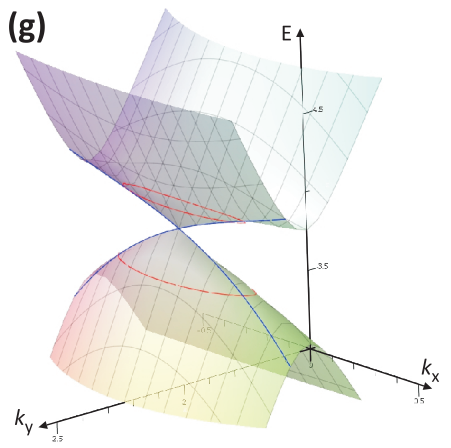

FIG. 6. Renormalization of the band dispersion due to the interaction of massive Rashba split bands. (a) Like in the present situation observed in W(110) experiment, bands with opposite sign of the effective mass cross. (b) A Rashba splitting lifts spin degeneracy and in a projected bulk band gap (white rhombus) bands with opposite spin polarization appear. (c) In the perpendicular direction, the bands remain a priori massive. (d)-(g) Results of a model calculation (see Ref. [39]). (d) Massive bands at the intersection point plotted along $k_{x}$. (e) Renormalization due to interaction provides a linear term which in (f) the resulting renormalized dispersion outweighs the quadratic term for small $k_{x}$ values. (g) Three-dimensional plot of the distorted cone with Dirac-like dispersion.

\section{SUMMARY}

In conclusion, we observe on the W(110) surface a Diraccone-like state along the line from $\bar{\Gamma}$ to $\overline{\mathrm{N}}$. With the help of first-principles calculations, we discuss aspects in favor and against a topological protection of this surface state with the conclusion that it is protected by mirror symmetry without the need to refer to topological arguments. Our model, besides a sufficient strength of spin-orbit coupling, requires just the presence of a mirror plane as it can be found on many lowindex surfaces like fcc(111), (001), or hcp(0001) and explains with rather general applicability why in these cases the spin polarization and the dispersion can appear Dirac like.

\section{ACKNOWLEDGMENTS}

G.B. gratefully acknowledges computing time at the JURECA supercomputer from the Jülich Supercomputing Centre and valuable discussions with $\mathrm{D}$. Wortmann. This work was supported by Impuls- und Vernetzungsfonds der HelmholtzGemeinschaft (Grant No. HRJRG-408).
[1] B. A. Bernevig and T. L. Hughes, Topological Insulators and Topological Superconductors (Princeton University Press, Princeton, 2013).

[2] Topological Insulators, edited by M. Franz and L. Molenkamp (Elsevier, Amsterdam, 2013).

[3] M. Z. Hasan and C. L. Kane, Colloquium: Topological insulators, Rev. Mod. Phys. 82, 3045 (2010).

[4] X.-L. Qi and S.-Ch. Zhang, Topological insulators and superconductors, Rev. Mod. Phys. 83, 1057 (2011).

[5] T. H. Hsieh, H. Lin, J. Liu, W. Duan, A. Bansil, and L. Fu, Topological crystalline insulators in the SnTe material class, Nat. Commun. 3, 982 (2012).

[6] P. Dziawa, B. J. Kowalski, K. Dybko, R. Buczko, A. Szczerbakow, M. Szot, E. Łusakowska, T. Balasubramanian,
B. M. Wojek, M. H. Berntsen, O. Tjernberg, and T. Story, Topological crystalline insulator states in $\mathrm{Pb}_{1-x} \mathrm{Sn}_{x} \mathrm{Se}$, Nat. Mater. 11, 1023 (2012).

[7] Y. Tanaka, Z. Ren, T. Sato, K. Nakayama, S. Souma, T. Takahashi, K. Segawa, and Y. Ando, Experimental realization of a topological crystalline insulator in SnTe, Nat. Phys. 8, 800 (2012).

[8] S.-Y. Xu, C. Liu, N. Alidoust, M. Neupane, D. Qian, I. Belopolski, J. D. Denlinger, Y. J. Wang, H. Lin, L. A. Wray, G. Landolt, B. Slomski, J. H. Dil, A. Marcinkova, E. Morosan, Q. Gibson, R. Sankar, F. C. Chou, R. J. Cava, A. Bansil, and M. Z. Hasan, Observation of a topological crystalline insulator phase and topological phase transition in $\mathrm{Pb}_{1 x} \mathrm{Sn}_{x} \mathrm{Te}$, Nat. Commun. 3, 1192 (2012). 
[9] L. Fu and C. L. Kane, Topological insulators with inversion symmetry, Phys. Rev. B 76, 045302 (2007).

[10] $\mathrm{L}$. Fu and C. L. Kane, Time reversal polarization and a $\mathrm{Z}_{2}$ adiabatic spin pump, Phys. Rev. B 74, 195312 (2006).

[11] D. Hsieh, Y. Xia, L. Wray, D. Qian, A. Pal, J. H. Dil, J. Osterwalder, F. Meier, G. Bihlmayer, C. L. Kane, Y. S. Hor, R. J. Cava, and M. Z. Hasan, Observation of unconventional quantum spin textures in topological insulators, Science $\mathbf{3 2 3}$, 919 (2009).

[12] D. Hsieh, D. Qian, L. Wray, Y. Xia, Y. S. Hor, R. J. Cava, and M. Z. Hasan, A topological Dirac insulator in a quantum spin Hall phase, Nature (London) 452, 970 (2008).

[13] M. Bianchi, D. Guan, A. Stróżecka, C. H. Voetmann, S. Bao, J. I. Pascual, A. Eiguren, and Ph. Hofmann, Surface states on a topologically nontrivial semimetal: The case of Sb(110), Phys. Rev. B 85, 155431 (2012).

[14] I. Aguilera, C. Friedrich, and S. Blügel, Electronic phase transitions of bismuth under strain from relativistic self-consistent GW calculations, Phys. Rev. B 91, 125129 (2015).

[15] F. Munoz, M. G. Vergniory, T. Rauch, J. Henk, E. V. Chulkov, I. Mertig, S. Botti, M. A. L. Marques, and A. H. Romero, Topological crystalline insulator in a new Bi semiconducting phase, Sci. Rep. 6, 21790 (2016).

[16] A. Varykhalov, D. Marchenko, M. R. Scholz, E. D. L. Rienks, T. K. Kim, G. Bihlmayer, J. Sánchez-Barriga, and O. Rader, Ir(111) Surface State with Giant Rashba Splitting Persists under Graphene in Air, Phys. Rev. Lett. 108, 066804 (2012).

[17] C. Pauly, G. Bihlmayer, M. Liebmann, M. Grob, A. Georgi, D. Subramaniam, M. R. Scholz, J. Sánchez-Barriga, A. Varykhalov, S. Blügel, O. Rader, and M. Morgenstern, Probing two topological surface bands of $\mathrm{Sb}_{2} \mathrm{Te}_{3}$ by spin-polarized photoemission spectroscopy, Phys. Rev. B 86, 235106 (2012).

[18] J. B. Pendry and S. J. Gurman, Theory of surface states: General criteria for their existence, Surf. Sci. 49, 87 (1975).

[19] A. Kobayashi, S. Katayama, Y. Suzumura, and H. Fukuyama, Massless fermions in organic conductor, J. Phys. Soc. Jpn. 76, 034711 (2007).

[20] M. Goerbig, J.-N. Fuchs, G. Montambaux, and F. Piechon, Tilted anisotropic Dirac cones in quinoid-type graphene and $\alpha$-(BEDT-TTF $)_{2} \mathrm{I}_{3}$, Phys. Rev. B 78, 045415 (2008).

[21] G. Montambaux, F. Piechon, J.-N. Fuchs, and M. O. Goerbig, Merging of Dirac points in a two-dimensional crystal, Phys. Rev. B 80, 153412 (2009).

[22] S.-M. Choi, S.-H. Jhi, and Y.-W. Son, Effects of strain on electronic properties of graphene, Phys. Rev. B 81, 081407 (2010).

[23] M. Trescher, B. Sbierski, P. W. Brouwer, and E. J. Bergholtz, Quantum transport in Dirac materials: Signatures of tilted and anisotropic Dirac and Weyl cones, Phys. Rev. B 91, 115135 (2015).

[24] T. L. Linnik, Photoinduced valley currents in strained graphene, Phys. Rev. B 90, 075406 (2014).

[25] A. A. Soluyanov, D. Gresch, Z. Wang, Q. Wu, M. Troyer, X. Dai, and B. A. Bernevig, Type-II Weyl semimetals, Nature (London) 527, 495 (2015).

[26] E. Rotenberg, J. W. Chung, and S. D. Kevan, Spin-Orbit Coupling Induced Surface Band Splitting in Li/W(110) and Li/Mo(110), Phys. Rev. Lett. 82, 4066 (1999).

[27] M. Hochstrasser, J. G. Tobin, E. Rotenberg, and S. D. Kevan, Spin-Resolved Photoemission of Surface States of $\mathrm{W}(110)-(1 \times 1) \mathrm{H}, \quad$ Phys. Rev. Lett. 89, 216802 (2002).

[28] A. G. Rybkin, E. E. Krasovskii, D. Marchenko, E. V. Chulkov, A. Varykhalov, O. Rader, and A. M. Shikin, Topology of spin polarization of the $5 \mathrm{~d}$ states on $\mathrm{W}(110)$ and $\mathrm{Al} / \mathrm{W}(110)$ surfaces, Phys. Rev. B 86, 035117 (2012).

[29] K. Miyamoto, A. Kimura, K. Kuroda, T. Okuda, K. Shimada, H. Namatame, M. Taniguchi, and M. Donath, Spin-Polarized DiracCone-Like Surface State with d Character at W(110), Phys. Rev. Lett. 108, 066808 (2012).

[30] K. Miyamoto, A. Kimura, T. Okuda, K. Shimada, H. Iwasawa, H Hayashi, H. Namatame, M. Taniguchi, and M. Donath, Massless or heavy due to two-fold symmetry: Surface-state electrons at W(110), Phys. Rev. B 86, 161411(R) (2012).

[31] D. Kutnyakhov, S. Chernov, K. Medjanik, R. Wallauer, C. Tusche, M. Ellguth, S. A. Nepijko, M. Krivenkov, J. Braun, S. Borek, J. Minár, H. Ebert, H. J. Elmers, and G. Schönhense, Spin texture of time-reversal symmetry invariant surface states on W(110), Sci. Rep. 6, 29394 (2016).

[32] A. Kimura, E. E. Krasovskii, R. Nishimura, K. Miyamoto, T. Kadono, K. Kanomaru, E. V. Chulkov, G. Bihlmayer, K. Shimada, H. Namatame, and M. Taniguchi, Strong Rashba-Type Spin Polarization of the Photocurrent from Bulk Continuum States: Experiment and Theory for Bi(111), Phys. Rev. Lett. 105, 076804 (2010).

[33] E. E. Krasovskii and E. V. Chulkov, Rashba polarization of bulk continuum states, Phys. Rev. B 83, 155401 (2011).

[34] A. M. Shikin, A. Varykhalov, G. V. Prudnikova, D. Usachov, V. K. Adamchuk, Y. Yamada, J. D. Riley, and O. Rader, Origin of Spin-Orbit Splitting for Monolayers of Au and Ag on W(110) and Mo(110), Phys. Rev. Lett. 100, 057601 (2008).

[35] A. M. Shikin, A. A. Rybkina, A. S. Korshunov, Yu. B. Kudasov, N. V. Frolova, A. G. Rybkin, D. Marchenko, J. Sánchez-Barriga, A. Varykhalov, and O. Rader, Induced Rashba splitting of electronic states in monolayers of $\mathrm{Au}, \mathrm{Cu}$ on a W(110) substrate, New J. Phys. 15, 095005 (2013).

[36] D. Thonig, T. Rauch, H. Mirhosseini, J. Henk, I. Mertig, H. Wortelen, B. Engelkamp, A. B. Schmidt, and M. Donath, Existence of topological nontrivial surface states in strained transition metals: W, Ta, Mo, and Nb, Phys. Rev. B 94, 155132 (2016).

[37] A. Varykhalov, O. Rader, and W. Gudat, Structure and quantumsize effects in a surface carbide: $\mathrm{W}(110) / \mathrm{C}-\mathrm{R}(15 \times 3)$, Phys. Rev. B 72, 115440 (2005).

[38] A. Varykhalov, O. Rader, and W. Gudat, Origin of Au nanostructures on tungsten surface carbides, Phys. Rev. B 77, 035412 (2008).

[39] See Supplemental Material at http://link.aps.org/supplemental/ 10.1103/PhysRevB.95.245421 for the details of experimental geometry and for further results of theoretical calculations.

[40] J. P. Perdew, K. Burke, and M. Ernzerhof, Generalized Gradient Approximation Made Simple, Phys. Rev. Lett. 77, 3865 (1996).

[41] E. Wimmer, H. Krakauer, M. Weinert, and A. J. Freeman, Full-potential self-consistent linearized-augmented-plane-wave method for calculating the electronic structure of molecules and surfaces: $\mathrm{O}_{2}$ molecule, Phys. Rev. B 24, 864 (1981).

[42] C. Li, A. J. Freeman, H. J. F. Jansen, and C. L. Fu, Magnetic anisotropy in low-dimensional ferromagnetic systems: Fe monolayers on $\mathrm{Ag}(001), \mathrm{Au}(001)$, and $\mathrm{Pd}(001)$ substrates, Phys. Rev. B 42, 5433 (1990). 
[43] D. Gosálbez-Martínez, I. Souza, and D. Vanderbilt, Chiral degeneracies and Fermi-surface Chern numbers in bcc Fe, Phys. Rev. B 92, 085138 (2015).

[44] Angle-Resolved Photoemission: Theory and Current Applications, edited by S. D. Kevan (Elsevier, Amsterdam, 1992).

[45] S. Hüfner, Photoelectron Spectroscopy Principles and Applications (Springer, Berlin, 2003).

[46] H. Zhang, C.-X. Liu, X.-L. Qi, X. Dai, Z. Fang, and S.-C. Zhang, Topological insulators in $\mathrm{Bi}_{2} \mathrm{Se}_{3}, \mathrm{Bi}_{2} \mathrm{Te}_{3}$ and $\mathrm{Sb}_{2} \mathrm{Te}_{3}$ with a single Dirac cone on the surface, Nat. Phys. 5, 438 (2009).
[47] S. Chernov, K. Medjanik, C. Tusche, D. Kutnyakhov, S. Nepijko, A. Oelsner, J. Braun, J. Minár, S. Borek, H. Ebert, H. Elmers, J. Kirschner, and G. Schönhense, Anomalous d-like surface resonances on $\operatorname{Mo}(110)$ analyzed by timeof-flight momentum microscopy, Ultramicroscopy 159, 453 (2015).

[48] D. V. Vyalykh, A. M. Shikin, G. V. Prudnikova, A. Yu. Grigor'ev, A. G. Starodubov, and V. K. Adamchuk, Quantum-well states and resonances in thin single-crystal layers of noble metals on W(110) substrates, Phys. Solid State 44, 164 (2002). 